\title{
Fibromatosis gástrica: un tumor raro en una localización infrecuente. Reporte de un caso
}

\author{
Gastric fibromatosis: a rare tumor in an infrequent location. Case report
}

\author{
Carlos Pacheco-Molina1, Armando A. Baeza-Zapata², Óscar R. García-De León ${ }^{1}$, Héctor M. Delgado- \\ Cortés ${ }^{2}$, Tania L. Salazar-Islas ${ }^{1}$ y Eduardo Navarro-Bahena ${ }^{1}$ \\ 'Departamento de Cirugía General; ${ }^{2}$ Departamento de Gastroenterología y Endoscopia Digestiva. Hospital Universitario José Eleuterio González, \\ Monterrey, Nuevo León, México
}

\begin{abstract}
Resumen
La fibromatosis tipo desmoide es un tumor benigno de suma rareza con características localmente agresivas. Se desarrolla en especial en tejidos blandos por su origen en los músculos y las aponeurosis. El diagnóstico se establece por inmunohistoquímica con positividad para vimentina, B-catenina y en ocasiones para actina de músculo liso. El espectro clínico de presentación es amplio, y según este y la resecabilidad se ofrece el tratamiento. Presentamos el caso de una paciente con síntomas de obstrucción al vaciamiento gástrico secundaria a una lesión subepitelial en cuerpo con inmunohistoquímica concluyente para fibromatosis gástrica.
\end{abstract}

Palabras clave: Fibromatosis agresiva. Tumor desmoide. Tumor gástrico benigno.

\begin{abstract}
Desmoid-type fibromatosis is an extremely rare benign tumor with locally aggressive features. It is predominantly developing in soft tissues due to its origin in muscles and aponeurosis. The diagnosis is established by immunohistochemistry with positivity for vimentin, B-catenin and sometimes for smooth muscle actin. The clinical spectrum of presentation is wide, based on this and resectability the treatment is offered. We present the case of a patient with symptoms of gastric outlet obstruction secondary to a subepithelial lesion in the gastric body with conclusive immunohistochemistry for gastric fibromatosis.
\end{abstract}

Key words: Aggresive fibromatosis. Desmoid tumor. Bening gastric tumor.

\section{Introducción}

La fibromatosis tipo desmoide es un tumor benigno de origen mesenquimal extremadamente raro'. La incidencia estimada es de 2-4 casos por 1,000,000 de habitantes $^{2}$, y representa el $0.03 \%$ de todas las neoplasias ${ }^{3}$.

La primera descripción fue realizada por McFarlane en $1832^{2}$. El término "desmoide», de origen griego (desmos), significa parecido o similar a un tendón y fue designado en 1838 por Muller ${ }^{4}$. Desde entonces

\section{Correspondencia:}

*Armando A. Baeza-Zapata

Avda. Madero y Gonzalitos, s/n

Mitras

Fecha de recepción: 25-04-2020

C.P. 64460 , Monterrey, N.L., México

E-mail: armando-baeza@outlook.com
Fecha de aceptación: 06-06-2020

DOI: 10.24875/CIRU.20000393
Cir Cir. 2020;88(S2):75-78

Contents available at PubMed

www.cirugiaycirujanos.com

0009-7411/C 2020 Academia Mexicana de Cirugía. Publicado por Permanyer. Este es un artículo open access bajo la licencia CC BY-NC-ND (http://creativecommons.org/licenses/by-nc-nd/4.0/). 
ha recibido múltiples denominaciones que dificultan su nomenclatura, como fibroma en forma de ligamento abdominal, tumor en forma de banda, fibroma abdominal, hiperplasia fibrosa, tumor fibroso recurrente abdominal, fibromatosis agresiva, tumor desmoide ${ }^{5} \mathrm{y}$ fibromatosis profunda ${ }^{6}$, siendo los tres últimos los más utilizados en la literatura reciente. En 1994, la Organización Mundial de la Salud estableció que es un tumor fibroblástico diferenciado con características biológicas intermedias entre el tumor fibroblástico benigno y el sarcoma fibroso ${ }^{5}$.

Este tumor se origina de las estructuras musculares y aponeuróticas ${ }^{6}$, se localizan con mayor frecuencia en el tejido blando de las extremidades ${ }^{7}$ y solo alrededor de un $20 \%$ se encuentran dentro de la cavidad abdominal ${ }^{2}$.

Tiene un espectro clínico heterogéneo, desde un curso asintomático ${ }^{8}$ hasta presentar complicaciones como obstrucción gastrointestinal o isquemia intestinal $^{1}$, debido a que presenta un comportamiento localmente agresivo e infiltra los tejidos normales circundantes, pero sin capacidad de producir metástasis ${ }^{9}$.

Aunque la supervivencia no se ve afectada a largo plazo, las recurrencias son frecuentes y las secuelas tras el tratamiento pueden afectar la calidad de vida ${ }^{6}$.

\section{Caso clínico}

Mujer de 53 años con antecedente de hipertrigliceridemia en tratamiento con fibratos. Es referida por historia de 3 años de evolución de pérdida de peso y saciedad temprana, progresivamente se agrega plenitud posprandial y masa palpable en el epigastrio, motivo por el que se realiza una tomografía contrastada toracoabdominal que reporta una lesión homogénea de contorno bien delimitado en la pared del cuerpo gástrico, de $7.5 \times 5.7 \times 5.8 \mathrm{~cm}$ (Fig. 1). Se realiza endoscopia superior y se encuentra una lesión subepitelial umbilicada en la curvatura menor a nivel de la unión del cuerpo con el antro gástrico (Fig. 2); se complementa con sonografía endoscópica (Fig. 3), con hallazgo de una lesión sólida hipoecoica en la pared gástrica que se origina en la capa muscular externa, bien circunscrita, con medidas de $4.9 \times 4.3 \mathrm{~cm}$. Se toman biopsias por aspiración con aguja fina, con reporte de citología transprocedimiento de tumor fusocelular. Se decide el manejo quirúrgico por sospecha de tumor del estroma gastrointestinal (GIST, gastrointestinal stromal tumor) y por la sintomatología en ese momento (Fig. 4). Durante la intervención se

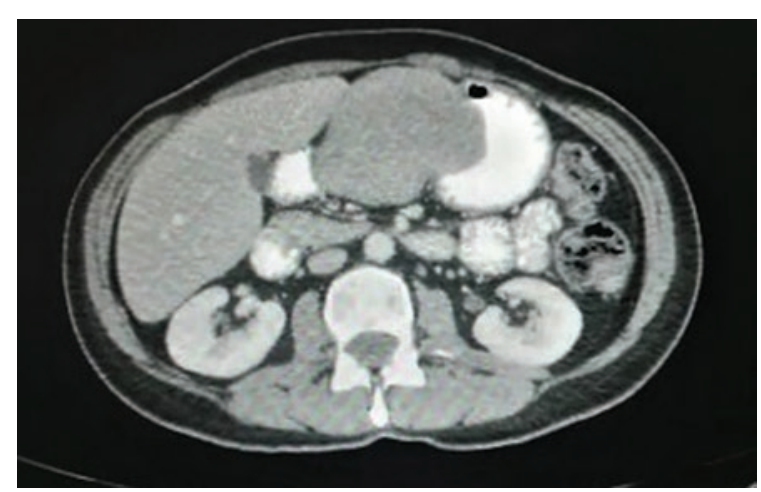

Figura 1. Tomografía simple toracoabdominal que muestra una tumoración a expensas de la pared del cuerpo gástrico.

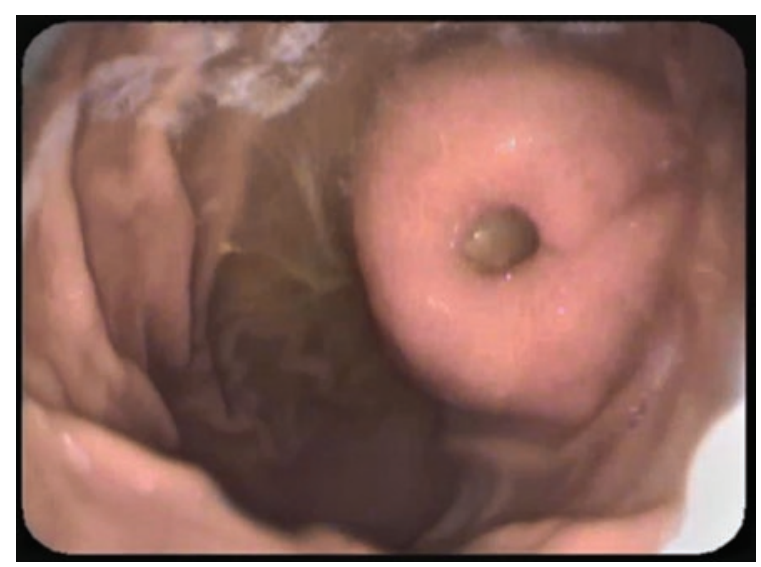

Figura 2. Endoscopia superior en la que se observa una lesión subepitelial umbilicada en la curvatura menor.

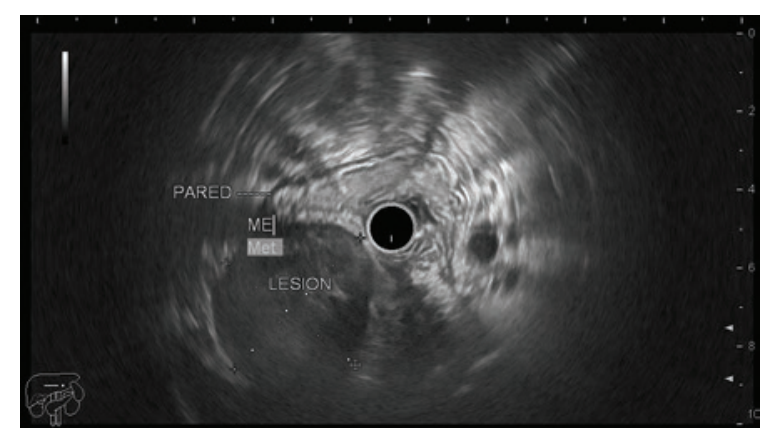

Figura 3. Sonografía endoscópica que muestra una lesión en el cuerpo gástrico, hipoecoica y de bordes definidos.

encuentra una tumoración firme en la curvatura menor que se extiende al antro gástrico. Se realizan gastrectomía distal (Fig. 5) y anastomosis gastroyeyunal en $Y$ de Roux, sin complicaciones durante el procedimiento. 


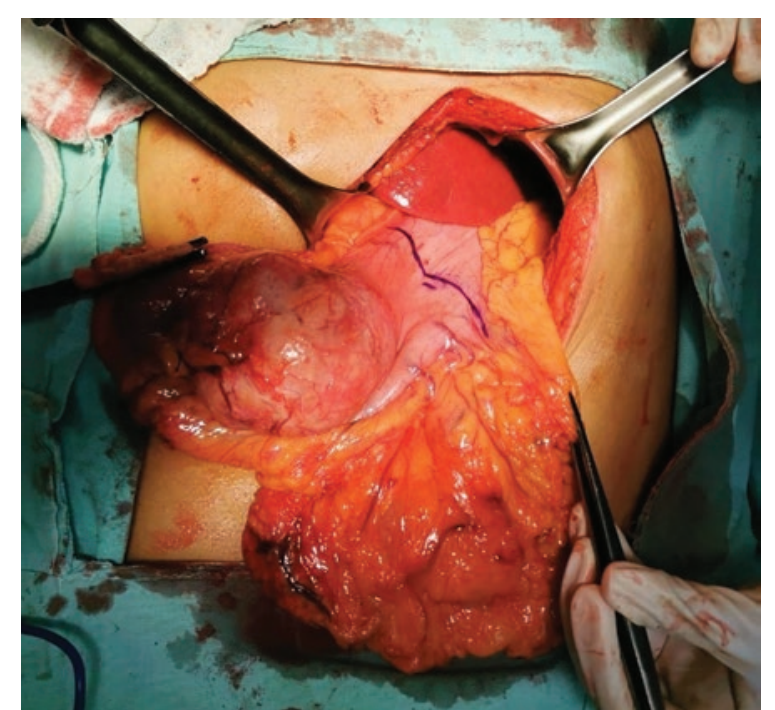

Figura 4. Exploración quirúrgica de la cavidad abdominal exponiendo la porción distal del cuerpo gástrico, el antro gástrico y el omento mayor.

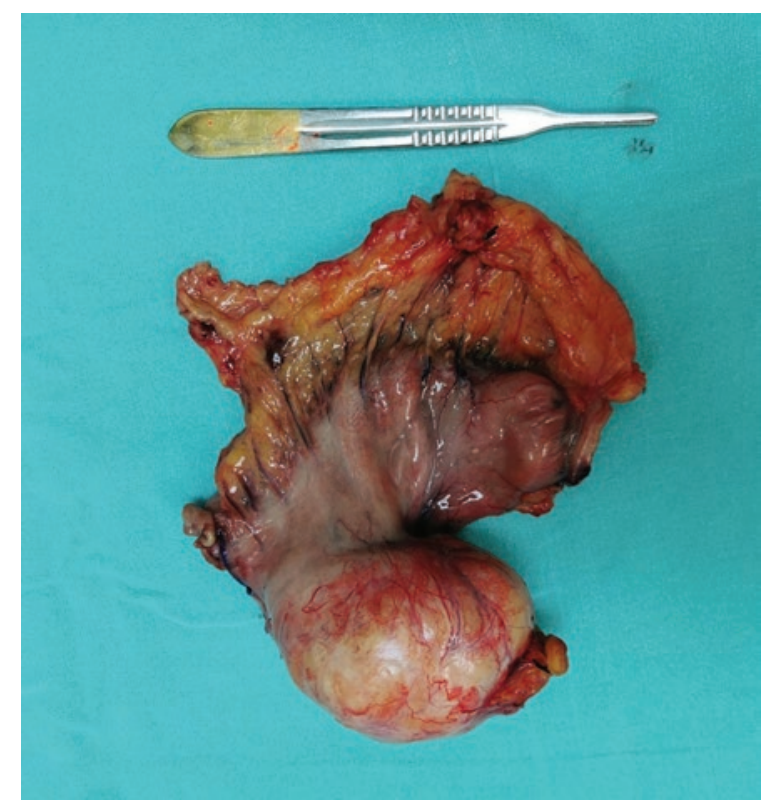

Figura 5. Resección quirúrgica de la porción distal de estómago con una tumoración firme en la unión del cuerpo y el antro gástricos.

El análisis histopatológico reporta tumoración en cuerpo gástrico, unifocal, con índice mitósico de 1 en $5 \mathrm{~mm}^{2}$, bordes quirúrgicos libres de neoplasia, con los siguientes resultados de inmunohistoquímica: CD117, DOG-1 y antígeno de músculo liso negativos, descartando GIST y leiomioma; B- catenina positiva en patrón nuclear y citoplásmico consistente para fibromatosis.
La paciente presenta una evolución clínica favorable $y$ permanece en vigilancia por el riesgo de recurrencia.

\section{Discusión}

El tumor desmoide (fibromatosis) es un sarcoma ${ }^{1}$ secundario a la hiperplasia anormal de fibroblastos o miofibroblastos en la profundidad de los tejidos blandos $^{5}$. La mayoría de los tumores son esporádicos ${ }^{3} y$ pueden localizarse en cualquier sitio anatómico, con predominio en hombros, pecho, pared abdominal, cabeza, cuello y muslos ${ }^{6}$. La minoría de los casos tienen predisposición genética'; el 15\% están asociados a poliposis adenomatosa familiar o síndrome de Gardner. En el síndrome de Gardner, el desarrollo de fibromas se considera una lesión precursora de un tumor desmoide, y cuando estos ocurren se localizan más comúnmente dentro de la cavidad abdominal ${ }^{4}$.

La causa de la enfermedad no está establecida, pero las agresiones físicas o quirúrgicas, trastornos endócrinos, estrógenos, radiación y anomalías cromosómicas se han considerado como posibles factores etiológicos ${ }^{1,5}$. La trisomía de los cromosomas 8 y 20 se ha reportado en múltiples casos, e incluso la trisomía 8 se relaciona con mayor riesgo de recurrencia'.

Los tumores desmoides se presentan típicamente entre los 15 y los 60 años de edad, con un pico de incidencia a los 30 años; son más frecuentes en las mujeres, principalmente después del embarazo, con disminución después de la menopausia, e incluso se ha visto que tienen un crecimiento más rápido en mujeres en edad fértil en comparación con los hombres ${ }^{6}$.

La presentación clínica es impredecible, desde una masa asintomática e indolente hasta una localmente agresiva con complicaciones relacionadas con obstrucción local e infiltración'.

Los estudios de imagen pueden ser de gran ayuda para complementar el diagnóstico y determinar la extensión de la enfermedad. La tomografía computada tiene limitantes al observarse la tumoración isodensa en comparación con el músculo estriado, con captación variable del contraste ${ }^{3}$. El estudio de elección que permite la mejor caracterización de estas lesiones es la resonancia magnética ${ }^{1}$ en secuencia T2W, en la que se observan bandas isointensas correspondientes a la disposición del colágeno ${ }^{3}$.

El diagnóstico definitivo es por histopatología, pues sin el apoyo de la inmunohistoquímica los tumores 
fusocelulares no pueden distinguirse entre sí. Microscópicamente se caracterizan por células fusiformes de apariencia uniforme con abundante colágeno, con núcleos típicamente pequeños, vesiculares, pálidos y bien definidos ${ }^{6}$. Las células tumorales son positivas para B-catenina y vimentina (en algunas regiones también se ha documentado este resultado para actina de músculo liso), y negativas para CKpan, EMA, proteína S-100, desmina, CD99, Bcl-2, ALK, CD34, CD68, CD163, CD21, CD23, CD117, DOG1 y receptores hormonales ${ }^{5}$. El $75 \%$ de los casos están asociados a mutaciones de B-catenina ${ }^{10}$, por lo que muestran positividad en la inmunohistoquímica en gran proporción de los casos; esta mutación es la más frecuente cuando están asociados con poliposis adenomatosa familiar ${ }^{6}$.

Los diagnósticos diferenciales incluyen el GIST, el pólipo fibroide inflamatorio, el schwannoma, el leiomioma, el tumor miofibroblástico inflamatorio, el sarcoma de células dendríticas foliculares, el histiocitoma fibroso maligno, el miofibroma y el tumor fibroso solitario 5 .

La dificultad para la erradicación definitiva de un tumor desmoide, asociado a su presentación clínica variable, ha llevado a la exploración de estrategias alternativas de tratamiento ${ }^{10}$.

El tratamiento dependerá de las características del tumor, que puede clasificarse en asintomático resecable, sintomático resecable, irresecable y recurrente. Al tratarse de una patología benigna, en los pacientes asintomáticos el tratamiento puede ser expectante. En los pacientes con síntomas y tumor resecable, la resección quirúrgica con márgenes negativos es el tratamiento de elección; en caso de márgenes positivos, la radioterapia ha sido benéfica.

En los tumores irresecables, en los que la cirugía implica un deterioro funcional, la radioterapia ha tenido resultados prometedores. La terapia sistémica está indicada cuando la terapia local ha fallado o está contraindicada; se utilizan antiinflamatorios no esteroideos, agentes hormonales (tamoxifeno), inhibidores de la tirosina cinasa y quimioterapia (doxorubicina, vinblastina, metrotexato), entre otros ${ }^{3,6}$.

\section{Conflicto de intereses}

Los autores declaran que no existe conflicto de intereses.

\section{Responsabilidades éticas}

Protección de personas y animales. Los autores declaran que para esta investigación no se han realizado experimentos en seres humanos ni en animales.

Confidencialidad de los datos. Los autores declaran que han seguido los protocolos de su centro de trabajo sobre la publicación de datos de pacientes.

Derecho a la privacidad y consentimiento informado. Los autores han obtenido el consentimiento informado de los pacientes y/o sujetos referidos en el artículo. Este documento obra en poder del autor de correspondencia.

\section{Bibliografía}

1. Rangunwala J, Sitta J, Prakash V, Vyas K, Roda M. Complex case of aggressive intra-abdominal desmoid-type fibromatosis status post cholecystectomy. Cureus. 2020;12:e7193.

2. Gómez Pedraza A, Padilla Rosciano A, Herrera Gómez A, León Takahashi AM. Tumor desmoide gigante de pared abdominal durante el puerperio tardío. Cir Esp. 2011;89:624-6.

3. Otero S, Moskovic EC, Strauss DC, Benson C, Miah AB, Thway K, et al. Desmoid-type fibromatosis. Clin Radiol. 2015;70:1038-45.

4. Lacka DE, Nasierowska-Guttmejer A. Fibromatosis - immunohistochemical evaluation, differential diagnosis from gastrointestinal tumors, and other mesenchymal tumours. Prz Gastroenterol. 2019;14:79-85.

5. Wang Y-K, Jiang B, Yang Y, Wang SN, Li YY, Meng NL, et al. Gastric aggressive fibromatosis: report of a case and review of the literature. Int J Clin Exp Pathol. 2019;12:372-7.

6. Martínez Trufero J, Pajares Bernad I, Torres Ramón I, Hernando Cubero J, Pazo Cid R. Desmoid-type fibromatosis: who, when, and how to treat. Curr Treat Options Oncol. 2017;18:29.

7. Mankin HJ, Hornicek FJ, Springfield DS. Extra-abdominal desmoid tumors: a report of 234 cases. J Surg Oncol. 2010;102:380-4.

8. Kummar S, O'Sullivan Coyne G, Do KT, Turkbey B, Meltzer PS, Polley E, et al. Clinical activity of the $\gamma$-secretase inhibitor PF-03084014 in adults with desmoid tumors (aggressive fibromatosis). J Clin Oncol. 2017;35:1561-9.

9. Wu C, Nik-Amini S, Nadesan P, Stanford WL, Alman BA. Aggressive fibromatosis (desmoid tumor) is derived from mesenchymal progenitor cells. Cancer Res. 2010;70:7690-8.

10. Crago AM. A prognostic nomogram for prediction of recurrence in desmoid fibromatosis. Ann Surg. 2013;258:347-53. 